\title{
TU/e EmonOWEN

\section{Real-time dynamics of the acoustically induced carrier transport in GaAs quantum wires}

\section{Citation for published version (APA):}

Alsina, F., Santos, P. V., Schönherr, H-P., Nötzel, R., \& Ploog, K. H. (2003). Real-time dynamics of the acoustically induced carrier transport in GaAs quantum wires. Physical Review B, 67(16), 161305-1/4. [161305]. https://doi.org/10.1103/PhysRevB.67.161305

DOI:

10.1103/PhysRevB.67.161305

Document status and date:

Published: 01/01/2003

\section{Document Version:}

Publisher's PDF, also known as Version of Record (includes final page, issue and volume numbers)

\section{Please check the document version of this publication:}

- A submitted manuscript is the version of the article upon submission and before peer-review. There can be important differences between the submitted version and the official published version of record. People interested in the research are advised to contact the author for the final version of the publication, or visit the $\mathrm{DOI}$ to the publisher's website.

- The final author version and the galley proof are versions of the publication after peer review.

- The final published version features the final layout of the paper including the volume, issue and page numbers.

Link to publication

\section{General rights}

Copyright and moral rights for the publications made accessible in the public portal are retained by the authors and/or other copyright owners and it is a condition of accessing publications that users recognise and abide by the legal requirements associated with these rights.

- Users may download and print one copy of any publication from the public portal for the purpose of private study or research.

- You may not further distribute the material or use it for any profit-making activity or commercial gain

- You may freely distribute the URL identifying the publication in the public portal.

If the publication is distributed under the terms of Article 25fa of the Dutch Copyright Act, indicated by the "Taverne" license above, please follow below link for the End User Agreement:

www.tue.nl/taverne

Take down policy

If you believe that this document breaches copyright please contact us at:

openaccess@tue.nl

providing details and we will investigate your claim. 


\title{
Real-time dynamics of the acoustically induced carrier transport in GaAs quantum wires
}

\author{
F. Alsina, ${ }^{1, *}$ P. V. Santos, ${ }^{1}$ H.-P. Schönherr, ${ }^{1}$ R. Nötzel, ${ }^{1,2}$ and K. H. Ploog ${ }^{1}$ \\ ${ }^{1}$ Paul-Drude-Institut für Festkörperelektronik, Hausvogteiplatz 5-7, 10117 Berlin, Germany \\ ${ }^{2}$ COBRA Inter-University Research Institute, Eindhoven University of Technology, 5600 MB Eindhoven, Netherlands
}

(Received 18 February 2003; published 29 April 2003)

\begin{abstract}
We have investigated the dynamics of the ambipolar transport of photogenerated electrons and holes induced by surface acoustic waves in GaAs quantum wires by using photoluminescence measurements with spatial and temporal resolutions. This technique allows us to trace the trajectory of charge packages in real time and to access the dynamics of radiative trapping centers. We identify two different kinds of trapping defects, which capture preferentially either holes or electrons.
\end{abstract}

DOI: 10.1103/PhysRevB.67.161305

PACS number(s): 73.50.Rb, 77.65.Dq, 78.47.+p, 78.55.Cr

In the past few years, new functions have been envisaged to store and to transport carriers employing the piezoelectric field of a surface acoustic wave (SAW). Light delay lines, for instance, have been suggested based on the ionization of photogenerated electron-hole $(e-h)$ pairs and transport of the resulting free electrons and holes in the maxima and minima, respectively, of the moving SAW potential $\Phi_{\mathrm{SAW}}{ }^{1}$ The periodic and dynamic distribution of carriers in the SAW piezoelectric potential opens new prospects for applications in quantum optics ${ }^{2}$ and quantum computation. ${ }^{3}$ These applications require the confinement of the $e-h$ pairs into narrow transport channels extending along the SAW propagation direction.

In this context, quantum wires (QWR's) become natural channels for carrier transport induced by SAW's. Previous investigations of the SAW-induced ambipolar transport in quantum wells (QW's) have shown an enhanced diffusion parallel to the wave front, which is inherent to the increase of the recombination lifetime due to the spatial separation of $e-h$ pairs by the piezoelectric field. ${ }^{4}$ The QWR confinement potential inhibits the lateral diffusion so that the carriers are then efficiently transported along the QWR axis as compact charge packages. ${ }^{5}$ Due to their small widths, onedimensional transport channels based on QWR's are preferred over those defined by etching techniques or by metal gates in applications where a dynamic and periodic spatial distribution or transfer of charge is required. ${ }^{6}$ Control of carrier occupation, in this case, is achieved through the frequency and amplitude of the SAW. Finally, the combination of QWR's and SAW's provides a mechanism for the formation of dynamic quantum dots if the scale of the SAWinduced spatial modulation becomes sufficiently small to induce quantum carrier confinement along the SAW propagation direction.

The understanding of the dynamics of the interaction between the SAW potential and photogenerated carriers, in particular, the mechanisms that control the acoustically induced transport in the one-dimensional channel defined by the QWR, is, therefore, of fundamental interest. A further relevant aspect regarding the transport in a one-dimensional system is how it is affected by the inhomogeneities and defects in the QWR. In this Rapid Communication, we address these questions by investigating the spatial distribution of photogenerated carriers as they are transported along the
QWR axis by the moving SAW field. The experiments, which are performed by detecting the photoluminescence (PL) with spatial and time resolutions, provide a means to track the charge packages in real time during the SAWinduced transport. The real-time detection provides insight into the transport dynamics, including the effects of radiative trapping centers. In particular, the experiments allow us to follow the processes of carrier capture into and emission from traps, as these are alternatively exposed to the electron and hole packages transported by the SAW. In this respect, the optical technique employed here is analogous to the electric transient spectroscopies used to investigate defects in semiconductors. We identify two kinds of radiative trapping centers with qualitatively different properties. The first one, which acts as a preferential trap for holes, is attributed to structural inhomogeneities along the QWR axis. The second one is a selective electron trap, which irradiates when exposed to a large hole concentration.

The semiconductor structures used in the present investigations consist of three stacked GaAs sidewall QWR's, ${ }^{7}$ each embedded in a QW. The QW's are separated by 10-nm-thick $\mathrm{Al}_{0.3} \mathrm{Ga}_{0.7} \mathrm{As}$ barriers grown by molecular-beam epitaxy (MBE) on patterned nominally undoped GaAs (311)A substrates. Each GaAs QW is $6 \mathrm{~nm}$ thick, the first one being located $120 \mathrm{~nm}$ below the surface. Prior to the MBE growth, 20-nm-high mesa stripes oriented along the [0 111$]$ direction were etched on the surface of the substrate. The formation of a fast-growing sidewall at the edge of the mesa with a convex unfaceted surface profile results in a vertical stack of quasiplanar QWR's, which are twice as thick as the QW's and have lateral extension $\approx 50 \mathrm{~nm}$. ${ }^{7}$ The quasiplanar nature of the sidewall QWR's makes them convenient for SAW's studies, since the propagation of the acoustic modes is not disturbed by topological structures on the sample surface. SAW's propagating parallel to the QWR's on the (311) surface were generated by aluminum split-finger interdigital transducers (IDT's) deposited on the sample surface. The IDT's were designed for operation at a wavelength $\lambda_{\text {SAW }}$ of $5.6 \mu \mathrm{m}$, corresponding to a frequency $\omega_{\mathrm{SAW}} /(2 \pi)$ of 533 $\mathrm{MHz}$ at the measurement temperature $T=12 \mathrm{~K}$. The amplitude of the acoustic field will be specified in terms of the linear power density $P_{l}$ defined as the acoustic power per unit length along the beam cross section. 


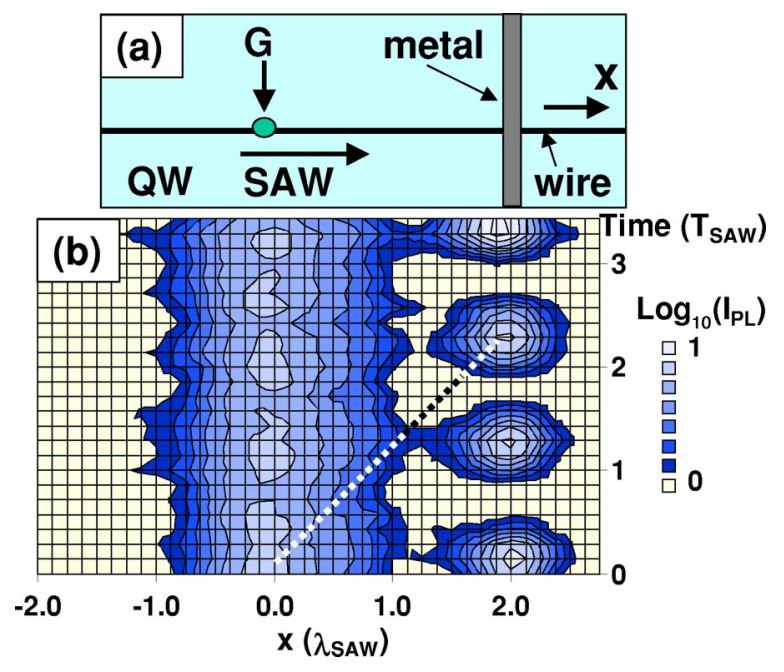

FIG. 1. (a) Setup for spatially and time-resolved PL measurements with SAW propagation along the QWR's. (b) Spatial (horizontal scale in units of SAW wavelength $\lambda_{\text {SAW }}$ ) and temporal (vertical scale in units of the SAW period $T_{\text {SAW }}$ ) dependences of the QWR PL generated by a microscopic laser spot under a SAW with $P_{l}=170 \mathrm{~W} / \mathrm{m}$. The dotted line indicates the trajectory of an electron package as it is transported by the moving SAW potential from the generation point $G$ to the edge of the metal stripe, where it recombines with transported holes.

The dynamics of the acoustically induced transport of photogenerated carriers was investigated by measuring the spatial and temporal evolutions of the PL collected along the QWR's, as illustrated in Fig. 1(a). The microscopic laser spot employed for PL excitation was placed on top of the QWR's in a position $G$ some micrometers away from a metal stripe deposited on the sample surface. PL excitation below the $\mathrm{Al}_{0.3} \mathrm{Ga}_{0.7} \mathrm{As}$ barriers was provided by the continuous radiation from a diode laser $\left(\lambda_{L}=680 \mathrm{~nm}\right)$. The metal stripe short-circuits the SAW piezoelectric field, thus forcing the recombination of the transported carriers. The PL emission along the QWR was imaged with a spatial resolution $\approx 2 \mu \mathrm{m}$ on a gated intensified charge-coupled-device (ICCD) camera. A time resolution of $0.5 \mathrm{~ns}$ was achieved by synchronizing the ICCD camera with the radio-frequency (rf) voltage employed to drive the IDT's.

Figure 1(b) displays the spatial (horizontal axis) and temporal (vertical axis) dependences of the integrated PL intensity in the presence of a SAW with $P_{l}=170 \mathrm{~W} / \mathrm{m}$. The spatial and temporal coordinates are expressed in terms of the SAW wavelength $\lambda_{\text {SAW }}$ and period $T_{\text {SAW }}=2 \pi / \omega_{\text {SAW }}$, respectively. The PL signal is modulated in time with the frequency of the SAW.

The study of the dynamics of the photogenerated carriers requires the knowledge of the absolute phase $\phi=k_{\mathrm{SAW}} x$ $-\omega_{\mathrm{SAW}} t\left(k_{\mathrm{SAW}}=2 \pi / \lambda_{\mathrm{SAW}}\right)$ of the SAW field relative to that of the PL. In previous studies on QW's, ${ }^{8,9} \phi$ was obtained by exploiting the fact that the type-I modulation of the band gap associated to the SAW strain field splits the excitonic PL line into a doublet. The oscillator strength of the doublet components is determined by the type-II modulation induced by
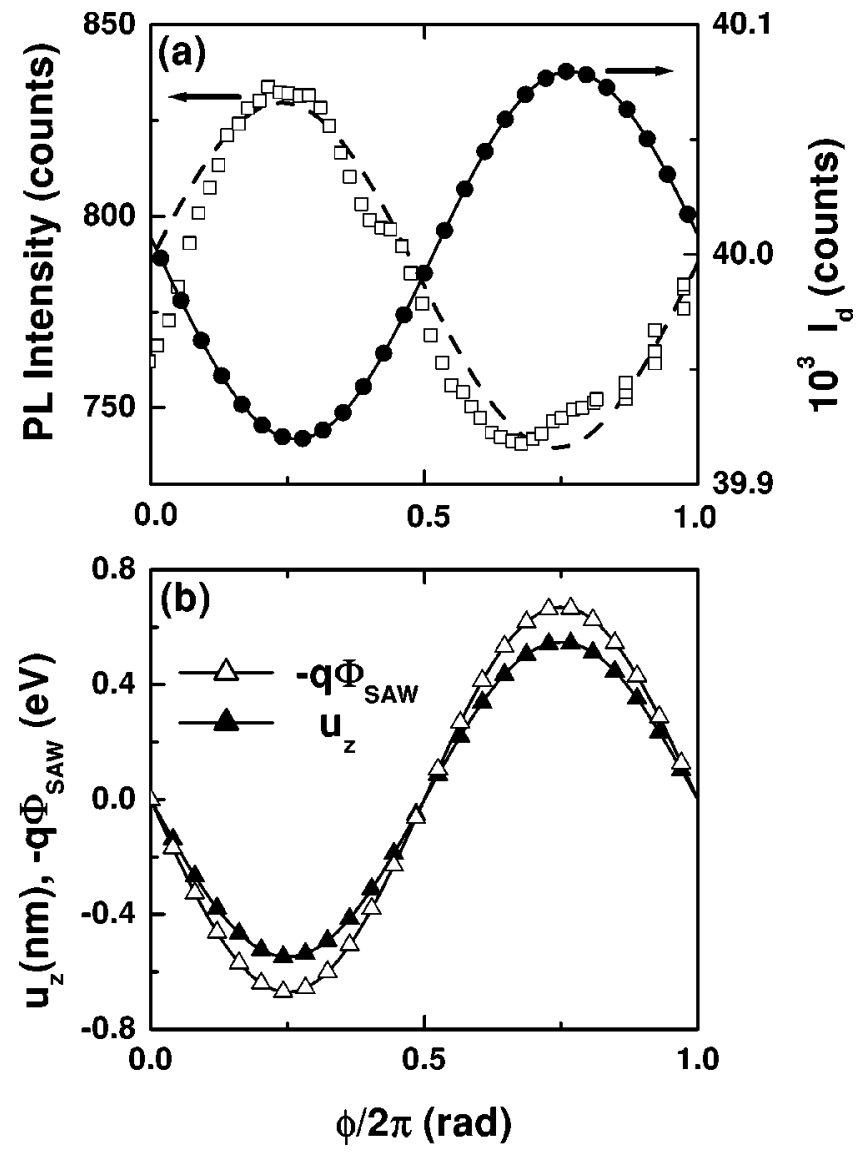

FIG. 2. (a) Time-resolved measurement of the PL (dashed line) and the interferometric trace $I_{d}$ (line with dots) recorded by a Michelson interferometer after a Fourier analysis that includes only the amplitude of the first harmonic. The raw data of the time-resolved PL measurement are also depicted (open squares) for comparison. (b) Calculated surface displacement $u_{z}$ and piezoelectric potential energy $-q \Phi_{\text {SAW }}$ induced by a SAW with a power density of $P_{l}$ $=100 \mathrm{~W} / \mathrm{m}$. The horizontal coordinates are scaled in SAW phase units $\phi=k_{\mathrm{SAW}} x-\omega_{\mathrm{SAW}} t$.

$\Phi_{\text {SAW }}$. The approach requires samples with PL linewidths small compared to the strain-induced splitting. This condition is not fulfilled for QWR's, where fluctuations of the confinement potential lead to energy variations that mask any effect of the strain field on the recombination energies. The alternative approach employed in the present measurements consists in detecting simultaneously the PL intensity and the surface displacement $u_{z}$ at $G$ as a function of time. $u_{z}$ was measured using a microscopic interferometer integrated in the PL setup, which employs an avalanche photodiode synchronized with the rf generator (time resolution $\approx 0.4 \mathrm{~ns}$ ) as detector. When the detection energy is switched from that of the excitation source to that of the PL line, the system directly yields the time dependence of the PL under the same experimental conditions.

Figure 2(a) compares time-resolved PL (open squares) and interferometric (line with dots) trace, which is proportional to $u_{z}$, measured in the presence of a SAW. It is interesting to note that the two quantities are phase shifted by $180^{\circ}$. In order to understand this phase relationship, we 
numerically calculated the acoustic and piezoelectric fields induced by the SAW along the [011] direction of the (311) surface. The modulation of the surface displacement $u_{z}$ and of the potential energy $-q \Phi_{\mathrm{SAW}}$ are displayed in Fig. 2(b). By comparison with Fig. 2(a), we conclude that the maximum PL intensity at $G$ coincides with the minimum piezoelectric potential energy, which concentrates electrons at the illumination spot. Therefore, the PL modulation in the QWR reflects the distribution of the electrons in the dynamic potential. Similar results were previously reported for (100) GaAs QW's, ${ }^{9}$ thus ascertaining the similarity of the carrier dynamics in the two cases.

The results of the previous paragraph allow us to identify the dashed line in Fig. 1(b), with slope $v_{\mathrm{SAW}}^{-1}$ and crossing the maximum PL at $G$, as the trajectory of minimum piezoelectric potential energy. This line also corresponds to the trajectory of the electron package as it is transported from $G$ to the recombination spot at the metal edge $M$. The amplitude modulation of the PL intensity is the strongest at $M$, where it drops below the detection limit during half of the SAW cycle. The strong PL modulation at $M$ results from the different transport properties of electrons and holes. Due to their higher mobility, the electron packages are highly concentrated close to the minima of $-q \Phi_{\mathrm{SAW}} .{ }^{9}$ The welldefined PL peaks at $M$ appear when these packages reach this position once in each wave cycle. The carrier dynamics in $G$ is more complex since it depends on several processes, i.e., the continuous nature of the carrier photogeneration, the subsequent ionization and extraction of the $e-h$ pairs from the generation area, and the screening of the piezoelectric field by the large density of photogenerated carriers.

Defects in the QWR induce trapping centers, which distort the carrier packages as they are transported. The SAW field in the measurements displayed in Fig. 1(b) is sufficiently strong to overcome the trapping potential so that the $e-h$ pairs are efficiently transported. In the presence of weaker SAW amplitudes, however, the effect of the traps becomes evident, as illustrated by the additional structures denoted by $D$ in the spatiotemporal profiles of Figs. 3(a) and 3(b). The defect in Fig. 3(a) traps the carriers and induces their recombination, as evidenced by the strong PL emission. No further transport is observed beyond this point, as indicated by the absence of PL emission near the metal edge $M$.

Additional information about the recombination site is obtained from the phase correlation between the PL and the SAW fields. The dotted line in Fig. 3(a) displays, as in Fig. 1(b), the trajectory of the electron package from $G$ to the recombination point. The strong recombination at $D$ coincides with the arrival of the electron package, thus indicating the presence of a trap for holes at this site. The trapping process, further referred as type $A$, is illustrated in Fig. 4(a). The trap initially captures holes as the hole density at site $D$ increases, when $-q \Phi_{\text {SAW }}$ reaches a maximum at this position. When the electrons arrive approximately half of a SAW cycle later (coinciding with a minimum of the potential energy), they recombine with the trapped holes. From the recombination dynamics in Fig. 3(a) we cannot exclude the possibility that the type- $A$ sites also trap electrons. The re-

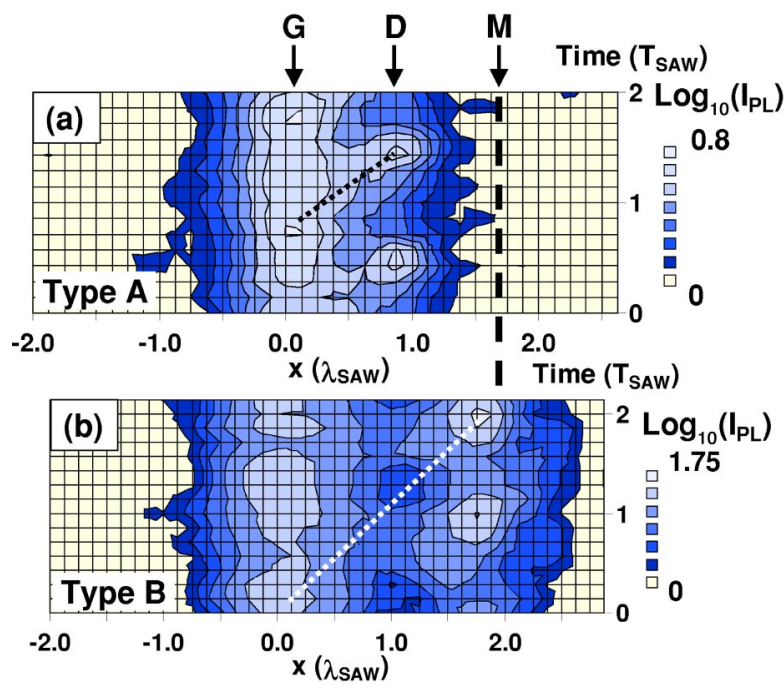

FIG. 3. Spatiotemporal PL profiles from two different QWR sections showing defect sites $D$ with carrier trapping properties of (a) type $\mathrm{A}$ and (b) type $\mathrm{B}$. The $\mathrm{SAW}$ intensities are (a) $P_{l}$ $=4 \mathrm{~W} / \mathrm{m}$ and (b) $P_{l}=92 \mathrm{~W} / \mathrm{m}$. The dashed vertical line indicates the position of the metal edge $M$. The units for the spatial (temporal) coordinates are given in terms of the SAW wavelength $\lambda_{\text {SAW }}$ (period $T_{\mathrm{SAW}}$ ).

combination dynamics of the type- $A$ defects is similar to that near a metal stripe, where the weakening of the SAW potential leads to the recombination of transported electrons and holes.

The measurements displayed in the contour plot of Fig. 3(b), which were recorded on a different QWR section, reveal a completely different trapping dynamics. The dotted line shows again the trajectory of the electron package. In contrast to the recombination near the metal edge and at the type- $A$ defects, an enhanced PL emission now occurs when holes cross the trap position. In fact, the PL intensity on these traps, which will be denoted as type $B$, reduces when they are exposed to electrons. This behavior allows us to unambiguously identify these recombination centers as electron traps. The electrons trapped during the minima of $-q \Phi_{\mathrm{SAW}}$

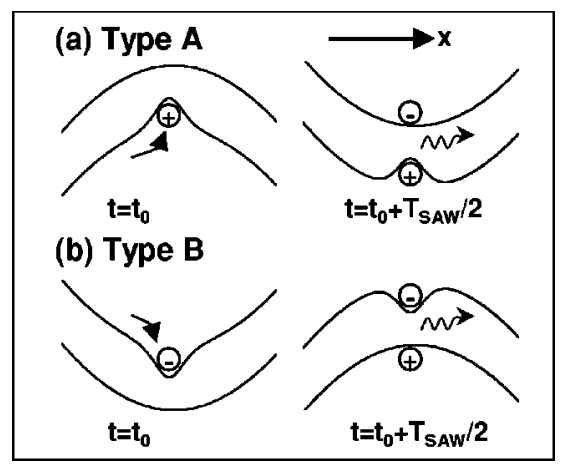

FIG. 4. Potential energy $-q \Phi_{\text {SAW }}$ distorted by the presence of either a (a) hole or an (b) electron trap. The arrows at $t=t_{0}$ represent the trapping of carriers by the defect. When the potential inverts at $t=t_{0}+T_{\mathrm{SAW}} / 2$, radiative emission takes place as carriers of opposite sign are brought to the position of the trapping site. 
recombine with holes transported to the trapping site half of a wave cycle later, thus leading to the enhancement of the PL after the passage of the electron package. This process of electron capture and recombination is illustrated in Fig. 4(b).

In thin nanostructures such as the QWR's investigated here, roughness at the atomic scale of the epitaxial GaAs/ $(\mathrm{Al}, \mathrm{Ga}) \mathrm{As}$ interface leads to a spatial distribution of quantum dotlike states: excitons localized at these states give rise to a characteristic PL spectrum composed of a series of sharp peaks distributed over a wide energy range. ${ }^{5,10}$ In addition, monolayer fluctuations in the QWR cross section cause the average potential to vary on the order of $10 \mathrm{meV}$ in a length scale of a micrometer. Weak SAW amplitudes, such as those generated in the measurements of Fig. 3(a), induce the delocalization of $e-h$ pairs on the length scale of the atomic roughness, as evidenced by the disappearance of the characteristic sharp peaks in the PL spectra (not shown). Longrange transport by the SAW potential can, however, be undermined by the potential barriers imposed by monolayer fluctuations. The energy difference of about $10 \mathrm{meV}$ between the PL signal detected at the generation position $G$ (higher) and at the position of the recombination site $D$ (lower) supports the assignment of the type- $A$ defects to monolayer fluctuations in the wire cross section. As the SAW intensity rises, the probability of carrier escape from the monolayer-related confinement potential increases and, eventually, the obstacle to SAW-induced transport disappears.
The potential inhomogeneities resulting from monolayer fluctuations affect simultaneously electrons and holes, and can, therefore, not explain the preferential electron trapping of the type- $B$ defects [cf. Fig. 3(b)]. Moreover, contrary to the type- $A$ case, PL spectra recorded at the type- $B$ defect site do not exhibit any particular energy shift as compared to the surrounding regions. In addition to the structural inhomogeneity discussed above, other sources of selective electron traps such as charged impurities and lattice imperfections should be considered. Further investigations are necessary to clarify the origin of these traps.

In conclusion, we have investigated the mechanisms involved in the SAW-induced ambipolar transport of photogenerated carriers along QWR's using spatially and timeresolved PL. The technique traces the position of the carrier packages in real time and allows us to access the role of trapping centers on transport. We identified two kinds of recombination centers with qualitatively different properties regarding the trapping dynamics. The first one captures preferentially holes, while the second one is a selective electron trap.

We thank H. Grahn for comments and for a critical reading of the manuscript. Support from the Deutsche Forschungsgemeinschaft (Project No. SA598/2-1) is gratefully acknowledged.
*Email address: falsina@pdi-berlin.de

${ }^{1}$ C. Rocke, S. Zimmermann, A. Wixforth, J.P. Kotthaus, G. Böhm, and G. Weimann, Phys. Rev. Lett. 78, 4099 (1997).

${ }^{2}$ C. Wiele, F. Haake, C. Rocke, and A. Wixforth, Phys. Rev. A 58, R2680 (1998).

${ }^{3}$ C.H.W. Barnes, J.M. Shilton, and A.M. Robinson, Phys. Rev. B 62, 8410 (2000).

${ }^{4}$ S.K. Zhang, P.V. Santos, and R. Hey, Appl. Phys. Lett. 80, 2320 (2002).

${ }^{5}$ F. Alsina, P.V. Santos, H.-P. Schönherr, W. Seidel, K.H. Ploog, and R. Nötzel, Phys. Rev. B 66, 165330 (2002).
${ }^{6}$ C.L. Foden, V.I. Talyanskii, G.J. Milburn, M.L. Leadbeater, and M. Pepper, Phys. Rev. A 62, 011803(R) (2000).

${ }^{7}$ R. Nötzel, M. Ramsteiner, J. Menninger, A. Trampert, H.-P. Schönherr, L. Däweritz, and K.H. Ploog, Jpn. J. Appl. Phys., Part 2 35, L297 (1996).

${ }^{8}$ T. Sogawa, P.V. Santos, S.K. Zhang, S. Eshlaghi, A. Wieck, and K.H. Ploog, Phys. Rev. B 63, 121307(R) (2001).

${ }^{9}$ F. Alsina, P.V. Santos, R. Hey, A. García-Cristóbal, and A. Cantarero, Phys. Rev. B 64, 041304(R) (2001).

${ }^{10}$ F. Intonti, V. Emiliani, Ch. Lienau, T. Elsaesser, R. Nötzel, and K.H. Ploog, Phys. Rev. B 63, 075313 (2001). 